\title{
ZUR KRITIK VON MEISTER ECKHART.
}

Spamer hat oben s. 307 ff. gezeigt, wie schlecht es um die handschriftliche beglaubigung Eckhartscher texte bestellt ist, und aus seiner Giessener dissertation über die zersetzung und vererbung in den texten der deutschen mystiker geht hervor, dass das zeugnis der rückverweisungen, aus denen Pahncke in seinen untersuchungen $z u$ den deutschen predigten meister Eckharts wẹtgehènde folgerungen gezogen hat, von sehr zweifelhaftem wert ist. Aber die hand, die niederriss, hat auch wider aufgebaut. Auf grund eines glücklichen fundes konnte Spamer dartun, dașs unter den 'tractaten', die uns Pfeiffer als erzeugnisse meister Eckharts vorgelegt hat, der fünfte mit sicherheit für Eckhart in anspruch genommen werden darf.

Glücklicherweise ist dieser fünfte tractat ziemlich umfangreich; so wird man hoffen dürfen, durch vergleichung des inhalts und der form ein urteil über die echtheit anderer erzeugnisse zu gewinnen, die zu dem namen Eckhart in beziehung gebracht worden sind. Ich glaube, einen beitrag zu solcher prüfung liefern zu können, indem ich das auftreten gewisser formaler eigentümlichkeiten verfolge.

In einer ausführlichen untersuchung zur technik der mhd. dichtung (Beitr. 30,431) habe ich dargetan, dass unbefangene menschliche rede ganz allgemein die neigung zeigt, dasselbe zweimal zu sagen, entweder in genauer widerholung des bereits ausgesprochenen oder 'mit ein bischen andern worten'. In jener abhandlung habe ich meine aufmerksamkeit auf die widerholte verkörperung ganzer gedanken gerichtet. 'Die erscheinung begegnet aber nicht minder auch bei der widergabe einzelner begriffe, vgl. F. Wenzlau, Zwei- und dreigliedrigkeit in der deutschen prosa des 14. und 15. jahrhunderts, Halle 1906, 
und meine besprechung dieser schrift, die demnächst im Literaturblatt erscheint, sowie Denifle, Taulers bekehrung s.89. Gar gerne wird dem einzelnen wort, zumeist mit hilfe einer conjunction, ein synonymon beigesellt, oder ein wort, das mit dem andern sich wenigstens teilweise deckt, seinen vorstellungsgehalt erweitert oder verengt. Beides, die satzwiderholung wie die wortwiderholung, findet sich auch in der literatur, die meister Eckhart zeitlich und sachlich nahesteht. Ich gebe zur veranschaulichung die beispiele von bestimmten abschnitten einiger mhd. prosadenkmäler (Wenzlau hat nur die übersetzer berücksichtigt):

Berthold von Regensburg II 150-164: 150, 18 daz er liuget und im nihtes nibt ernst ist und im nindert umbe daz herze ist. - 151, 22 der ist vil und vil, wunder und wunder. - 152,9 der sünden wirstiu und aller sünden unreinestiu. - 153,10 tuot eht wol und wol. - 153, 32 lonet mit übergender maze von freuden und mit gehufter maze der ewigen freude. - 154,3 wir sin geladen zuo den ewigen freuden und zuo der ewigen wirtschaft. - 154, 7 also groz wunne und ere und freude ist da. - 154, 22 man ist gar schiere und gar snelle uf dem selben wege. - 155, 5 so heizet der enge (wec), der smal. - 155, 36 sie trahten und leiten alle ir sinne dar an, wie...- 156,15 tet unser herre den selben wec zuo und hat in verrunet. - 157 kein beispiel. - 158,15 sin geladen zuo der ewigen freude, zuo der ewigen wirtschaft. - 158,19 daz sint arme liute und dïrftige. - 158, 20 die lident groze marter und armuot (von hunger und von froste). - 158,38 mit gebete, mit venie. - 159, 9 der waere als jaemerlich und also griulichen. - 159,12 hoeren ir not und ir kumber. $159,34 \mathrm{ez}$ ist sin gelerter und laufent dort hin und ist sin spot. 159, 39 die da sinnic sin und den got rehter sinne verlihen habe. $-161,4$ daz sie ez erfunden und rieten. - 160 kein beispiel. - 161,30 daz du von ersten erfunden und geraten hast. - 162 kein beispiel. - 163, 10 versmaehen mit nide und mit hazze. - 164, $17 \mathrm{daz}$ sie zuo dem himelriche komen ze grozem lone und ze grozen freuden.

Altdeutsche predigten, hsg. von Schönbach, I 100-114: 100, 22 daz ir kein vorneme noch enwizze. - 100, 32 sint dar inne trachen und lintwürme, natern und slangen und aller slahte bose und übil gewürme. - 101,31 bezeichent die offene bekorunge und anevechtunge. - 102,17 der phlach riches und gutes gewandes. - 102,19 was suchtich und sere. - 102, 22 do unser herze do lonen und ergetzen wolde den armen siner not und siner quale. - 102,31 wane ich grozer pine und quale liden. 102,34 hatte da wider alle ungenade und ungemach, nu hat er trost und genade und du must da wider ungemach und ungenade haben. - 103,10 daz ir uwir almusen gerne gebit und der armen niht vorgezzet. - 103, 29 enbesliezit uwir herze niht vor der barmunge und envorhartit so niht, so ir entut genade und ettelichen trost. - 104,37 clagete und weinte vil 
bitterliche. - 105, 7 můze uch helfen und gesterken zu allin gutin dingen. - 105, 16 der machte ein groz imbiz und eine groze wirtschaft. - 105, 38 newart kein spise noch kein trank so suze noch so gut. - 106, 4 der uns da ladet und uns heizzet cumen. - 106,19 swer die zu sunden und zu bosheit keret. - 106, 29 get iz an die untruwe', an daz vorretnisse. 107,1 den got alsus twingit und gestingit. - 108, 18 hiran sult ir vlizclichen und dicke gedenken. - 109, 28 bose und unrehte lute. - 110, 12 uf ime gebnwit und gestetigit ist. - 110,30 daz ist mir leit und zorn. 110,36 uwere sele zu troste und zu genaden. - 111, 6 ir gerechticheit und ir gute enwere manichvaldiger und grozir. - 111, 12 da bie retit und gebutet uns unser herre. - 111, 19 sin herze was vol nydes und hazzes. 111, 26 wanne iz lutir und reine was. - 111,37 sach sine bose gedanken und sinen bosin willen. - 112,16 daz ir rehte und redeliche lebit. 112, 17 vliht daz geschelde und idewize. - 112,34 die kirwie und kirchmesse die wurden gesazt unserm herrin zu lob und zu erin und euch zu troste und zu genaden. - 113 kein beispiel. - 114,4 daz er wol dutin und uz legen kan. -- 114, 24 da enist kein gedoze, da ist iz stille. 114,26 in dem gebete, in den veinen.

Deutsche mystiker, hsg. von Pfeiffer. I 6-29 (Hermann von Fritslar): 6 kein beispiel. - 7,7 der durch iren willen gemarteret und gekruziget ist. - 7,16 daz daz volg sich ubele hate unde betrubet waren. - 7,19 do vant her sente Matheum inne gevangen und in in den kerker geleget. - 8,4 gingen si vrilichen vor fursten und vor herren. - 8,11 habe gedult unde wis in vride. $-8,32$ wan her ein furste was der kristenheit und ein houbet. $-9,27$ der himel ist veste an im selber und enmag nicht keinen vremeden indrug inphan. - 9,28 was veste und stete an deme glouben. - 9, 32 der do ein rein kusch man was. - 9, 32 hate sente Andreas gelobet zu dinen und gekorn zu eime aposteln. - 10,5 mir rates und mich ane wises. - 10,6 vil swinder und behender wort sprach si. - 10,19 inprant wart in boser begerunge und in vile bekorunge zu der jungfrowen. - 11,12 fur her sinen weg und verswant. - 12, 30 liz alle ire wunden vornuwen und offenen. - 13,25 deme ich mich gegeben habe und deme so han ich mich vertruwet. - 13,26 den soldest du billichen anebeten und soldest in eren. - 14, 2 werltliche wise und werltliche bilde. - 14, 9 di dises irdenschen gutes nit begeren noch nit enwollen. - 14, $30 \mathrm{daz}$ si sie liplichen trug und gebar. - 15, 3 alle di heimlichkeit und alle di innekeit. - 15, 13 dar umme wer her der beste meister gewesen oder schriber. 15,17 iz zu aller ersten geware werde oder gevule. $-16,5$ welich di geiste sin oder die sele. - 16,9 gen mit grozzen dingen umme und bekummern sich nit mit kleinen dingen. - 16,19 dise jungfrowe ensolde nicht verlazen sin noch verworfen von keinem manne. - 16,27 der kuntschaft hat aller geistlichen ubungen nnd aller geistlicher werken. - 16, 30 bedutet luter abgescheidenheit von aller dirre werlde und von gebrechlichkeit dirre dinge. - 16;35 di unberuret blibet und unbetrubet von allen widerwertigen dingen. - 16, 38 in der gnade gotis bekliben und gewurzelet sin. - 16,39 der dises kindes pleger und huter was. - 17, 12 di der sele gehelfen muge oder si bereiten muge, daz daz ewige wort in ir geborn werde. - 17,28 
von dem munde des obersten oder des aller hohesten. - 18,9 benam den aneval der erbesunden und vertilgete di. - 18,17 di enweiz des nit vur war und genzlichen. - 19, 2 so ich aller schonest und aller klerest mochte. - 19,10 hate ez eweclichen ane gesen und vorgesatzit daz her der erste solde sin. - 19,25 enhat kein gesetze noch gebot dar uber. - 20 kein beispiel. - 21,23 ein iclich kusche mensche ist ein tempel des heiligen geistes und der heilige geist wonet in im. - 22, 10 swanne diz der vernunft erschinet und ir geoffenbarit wirt. - 22,12 wille und vernunft sinken und fulen des ewigen wortes, daz der vater in in gebirt und offinbart. - 22, 18 swanne di sele dise stucke bedenket und betrachtet mit innekeit und mit andaht.

Es bieten also: Berthold auf 3 seiten kein beispiel, auf 6 seiten je eines, auf 3 seiten je $z w e i$, auf 1 seite drei, auf 2 seiten vier beispiele; die altdeutschen predigten einmal kein beispiel, 5 mal je eines, 5 mal je drei, 2 mal je vier, einmal sechs und einmal acht beispiele; Hermann von Fritslar 2 mal kein beispiel, 3 mal je eines, 3 mal je zwei, 8 mal je drei, je einmal vier, fünf und acht beispiele.

Ganz anders liegen nun die verhältnisse in dem tractat, den Spamer für meister Eckhart gesichert hat. Da das denkmal ziemlich umfangreich ist (s. 419-448 bei Pfeiffer), gebe ich das material nur für einzelne herausgegriffene seiten:

419, $8 \mathrm{daz}$ den menschen anvallet unde trenget. - 419, 10 an swacheit, an ungemach und an smerzen des libes. - 419,13 sich troesten mag in allem sinem ungemache, betrüepnisse unde leide. - 419, 14 hat man etliche warheit dar uz unde da von genomen. - 419,15 daz den menschen billichen unde genzelichen getroesten mag. - 419,16 dar nach vindet man bi drizic sachen unt lere (in der der mensch sich tröstet). - 419, 18 vindet man bilde unt lere. - 419,19 an worten und werken, die wise liute hant getan unde gesprochen. - 419,24 diu güeti ist niht gemachet noch geschaffen noch geboren. - 419,25 si ist gebernde unt gebirt den guoten. 419,26 ist ungemachet, ungeschaffen. - 419, 26 unt doch geboren kint unde sun der güeti. - 419,33 in dem guoten ist alleine ein wesen, ein leben. - 419, $34 \mathrm{daz}$ nimet er beidiu von der güeti unt in der güeti. 419,35 da von ist unt lebet unt wonet er. - 419, 37 würket er mit der güeti, in der güeti. und diu güeti mit ime und in ime. - 423,5 allez ungemach unt schade ist niht sunder gemach. - 423, 7 daz gotes triuwe unt sin eigen weslichiu güeti enlidet des niht. - 423, 8 daz kein leit unde bekerunge unlidelicheu unt ze überswenclichen si. - 423, 9 er git unt machet alle zite etwaz trostes. - 423, $11 \mathrm{daz}$ luter boes oder leit müge sin noch gewesen. - 423, 15 so belibet er ungetrost unt riuwic. - 423, 16 getroestet sin unt ane leit. $-423,16$ der sich keret zuo dem schaden unt leit. - 423, 17 (bildet daz in sich) unt siht dar in und sihet daz an mit leide. 423, 21 widerbildeti sich in die sehzic unt sehi die an. - 423, 24 daz min 
niht ist unt mir verloren ist, daz muoz leit unt untrost geben unde betrüepnus. - 423, 26 sprichet Salomon: 'in den tagen der leide unde der betrüebede vergiz niht der tage der güeti unt der lüsten.' - 423, 28 so du bist in leide unt in ungemache, so gedenke des guoten unt des gemaches. - 423, 34 ist er siech und in grozem smerzen sines libes. 427, 3 also nime ich enbern unde niht nemen. - 427,6 war umbe der mensche fro si unde getroestet. - 427,7 so vindet noch weiz man niht, des man sich froüwe, denne got unt gotes willen alleine. - 427,11 lidet dur got unt gotes willen. - 427,15 so hàt er vor gote unt in gote sich behalten allez daz, darumbe er den schaden oder daz leit niht liden wölte. - 427, 22 lịden wellen gerne unt gedulteclichen. - 427, 24 (als wir ouch sehen in der nature) daz ein mensche schoener unt künstericher ist denne der ander. - 427,26 daz ein guot mensche wol mac ein guot mensche sin und doch berüeret werden unde wanken von natiurlicher liebi zuo vater unt muoter. - 427. 28 nach dem er ist guot unt bezzer - 427, 29 berüeret wirt unt gewar wirt natiurlicher minne unde neigunge zuo vater unt muoter. - 427, 36 so were im gar sere rehte unt würde sicherlich getroestet in leide. - 427, 40 möhte der diep werlichen, genzlichen unt luterlichen, willeclichen unt froelichen den tot liden. - 431, 3 dar zuo unt darnach saget ez me und ist sneller und ist ime sin louf sijezer unt wunneclicher. - 431, 7 gelicher dem, daz ez jaget, dar zuo ez ilet. - 431, 8 ziuhet und locket von der kraft. - 431,9 dar umbe gestillet noch benüeget niht noch dem ... noch dem ... - 431, 15 enbrant und entzündet wirde. - 431, 18 der rede unt dirre wäheit han wị ein offen urkünde unde bewisunge. 431, 19 wenne daz fiur an gezündet unt enbrennet daz holż. — 431, 23 doch gestillet noch benüeget noch geswiget nimmer niht weder holz noch fiur an keiner wermi noch hitze. - 431, 25 unz daż daz fiur gebirt sich selber in daz holz unt git ime sin eigen nature und ouch ein wesen sin selbes. - 431, 29 ein rouch, ein widerkriec, ein krasp̉len uñd ein strit zwischent furre unt holze: - 431,30 wirt benomen und hine getan. 431, 33 in ime treit den underscheit und zweiunge. - 431, 35 als der munt in dem unt an dem wine minnet und suochet den smak unt die süezikeit. - 431, 40 hazzet und niht minnet gelichnüs. - $-435,4$ ez ist ein fürganc unt verlust lidennes dur got. - 435, 8 geliten han ist verre unt ungeliche lidenne. - 435,11 umbe daz gotes ere unt lop gemeret unt gebreidert würde. - 435, 16 von gote wölte gesunderot unt gescheiden sin. - 435, 17 von gote sich scheiden oder sunderon wölte. - 435, 22 ist daz inre werc dar nach götlich unt gotvar. - 435, 31 nimet unt schöpfet allez sin wesen ni ergen denne von gote unt in gotes herzen. - 435,35 uz getragen unt uz gegozzen in einen niderval der gotheit. - 435, $37 \mathrm{daz}$ allez unt dem geliche unt ouch gelichnüs selben gote verre unt frömde sint. - 435, 39 sie haftent unt behaftent unt gestillent in dem. - 439, 2 als man wenet oder gemeinlichen sprichet. - 439,3 ez ist ein geloube unt ein. götlichiu lere. - 439, 4 sin leben wunneclichen unde froelichen wirt. - 439, 12 blibende in des vaters schoze unt herzen. - 439,16 der in des vaters schoze unt herzen ist. - 439, 20 alle frömdi unt verre hazzet got. - 439, 20 zuo ein locket unt ziuhet got. - 439, 22 gezogen werden über nature unt über- 
bildet. - 439, 27 daz si zerbreche oder verderbe unt ouch enrüere. - 439, 32 den menschen bezzer machen unt im lieber tuon möhte. - 443,1 muoz verlieren sin bitterkeit unt luter süez werden. - 443,2 e denne ez des menschen herze iemer müge bewegen noch berüeren. - 443, $7 \mathrm{ez}$ wirt allez verbrant unde verderbet. - 443,18 ouch sol der mensche bekennen unde wizzen. - 443, 23 daz got wider ime vindet unt ime ungelich ist. 443,24 daz ist leit, untrost unt bitterkeit. - 443, 25 daz got mit uns ist in lidenne unde mitelidet mit uns. - 443,37 minnecliche unt miltecliche so lident dur got. - 443,38 sit daz ez so groezlichen nütze ist und seleclichen. - 447,2 ir leben hant gegeben unt ir selbes willeclichen verzigen. - 447, 4 tugende machent unmüglichiu dinc mïglich unt ouh likt unt süeze. - 447,9 unt enthielt sie unt mante sie sunderlichen alle dar zuo, daz si niht erschreken. - 447,13 solte sich gar übele unt ouch groezlichen schamen. - 447, 20 waget guot, lip unt sele dur zergancliche unt ein kurze ere. 447,36 ist der stap in sich selben reht unt niht krumb. - 447, 38 wer allerlei liphaftikeit und bilden inne bekennet. - 447,40 der lachet unt spottet min.

Das sind der reihe nach $17,18,16,20,13,10,9,8$ beispiele auf die seite. Ich habe noch für andere seiten die beispiele gezählt und teile wenigstens die zahlen mit: s. 420 10 beispiele, 4219,424 10, $42516,43017,434$ 16, 437 8, $43819,44015,44211,44418,4459,4467$.

Man sieht, hier ist der allgemeine unbewusst geëbte brauch zum bewussten, in gesteigertem masse angewanten stilmittel geworden.

Im scharfen gegensatz zu tractat $V$ zeigt nun aber die grosse masse der übrigen tractate keine bewusste anwendung des stilmittels der widerholung; die zahlen gehen über den sonst üblichen durchschnitt nicht hinaus.

Tractat I (s. 373-382) enthält nur 29 beispiele:

373, 7 alliu vergenclichiu dinc sint mügelich unde lihte ze lazenne. - 373, 12 smehe unde bitter machent alliu vergenclichiu dinc. - 375, 22 alliu diu süezikeit unde wollust. - 375, 29 gebent sie groezer wollust unde süezikeit. - 375, 32 wolde gerne alle zit in der süezikeit unde luterkeit sin. - 376,11 ie si luterr wirt und unvermenget mit allen den dingen, diu ... - 376, 28 ie daz viur me materien hat, ie ez me verzert und in sich verwandelt mit grozer hitze. - 376, 33 daz die gedenke der geistlichen sachen lustliche unde vlizecliche würkent. 376,34 gesterket an guoten und an heiligen gedanken. - 376,36 daz der mensche sine gedenke nutzliche unde lustliehe inkeren möhte. - 376,38 so vil boezer und unnützer gedenke. - 376, 39 ie der mensche, der mer hat unde den me under lit. - 377, 3 in ein vergezzen kumet unde niht enahtet aller dinge. - 377, 12 aller götlichen vollekomenheit unde selikeit. - 377, 18 erhebet unde dur- 
schinet die sele. - 377,20 in aller heilekeit unde vollekomenheit des lebens. - 377,31 ie diu lust merer ist unde diu selikeit. - 378,14 an aller heilikeit und an aller vollekomenheit. - 378,18 heiligiu unde vollekomeniu werc. - 378, 28 alle vollekomenheit unde luterkeit. - 378, 29 alse geliutert unde vollekomen. -- 378, 34 luter und ungemenget von allem dem. - 379, 35 diu groezte vreude unde selikeit. - 380,10 vro in gemache und in ungemache, in liebe, in leit. - 380, 33 gegeben noch bewisen (die freude). 381,10 des ewigen lebens unde der selikeit. - 382,1 in der ordenunge und in der maze (=3). - 382, 26 den nutz unde die selikeit.

Der tractat II (s. 382-394) enthält aứ s. 383-387 4+ $4+3+0+5$ beispiele:

383,7 twingen mit vlize unde mit grozem ernste. - 383, $18 \mathrm{ez}$ nimet sunder glichnisse unde sunder bilde. - 383, 35 wie rehte ordenlich unde wie edellich ein iegelichiu kraft geordent ist unde gesetzet uf ir stat. 384,3 des ist si ledic unde vri. - 384,12 in dem liehte und in der edelkeit des gelouben. - 384, 26 daz verstentnisse wirfet sich uz unde hoeret unde vernimet. - 384, 27 dar umbe bescheidet ez und ordent unde setzet. - 385, 17 ez sprichet ein meister: got wirt getragen unde gesetzet in die sele. - 385, 27 so treit erz allererst unde setzet ez in daz gehügnisse. - 383, 27 also wirt got getragen unde gesetzet in die sele. - 387, 9 vindet sich unde bekennet sich creature. - 387,15 ir gotes aller besten vriunde sit und im aller heimlichest sit. - 387, 22 vliezent drie persone personlich unde wesenlich in daz wesen. - 387,40 ein sprechen sunder wort und sunder lut.

Der tractat III (s. 394-416) bietet auf fünf seiten $3+1$ $+3+2+4$ belege:

395,6 so wirt si unedel unde grob. $-395,26$ wider lebende ze werden und uf ze sten mit warer riuwe und buoze. - 400, 26 sint in ime als luter und edel. - 405, 1 als wenic got in namen und in worten mac begriffen werden. - 405, 2 alse wenic mac diu sele in bilden noch in formen begriffen werden. - 405, $8 \mathrm{daz}$ diu sele von nature zem himele beschaffen ist und daz got ir rehtiu erbestat ist. - 410,32 als ein fiurigiu nature, diu allez daz zuo ir ziuhet und in sich verwandelt, daz in si kumt. 410,33 so si diu sele zuo ir ziuhet und in ir verwandelt. - 415, 22 überwinden müge ir ansuochunge unde schedeliche lüste. - 415, 25 ze tuon unde ze halten allez daz du geboten hast. - 415, 32 minne unde lust, sie ze vollebringen. - 415, 35 uf menschliche ungewisheit unde krankeit.

\section{Der tractat IV (s. 416-418) enthält $2+3+3$ beispiele:}

416, 22 ist ez namlos und engat allen worten, diu creaturlich sint. 416, $26 \mathrm{daz} \cdot$ gelicher müge sin der götlichen art und im geliche widerblicket. - 417, 1 nach sinem endelosen bilde und ewigen bilde. - 417, 8 die sint in einformikeit so gelich und so nahe. - 417,13 nach allem irem grunde würkenlich und wesenlich. - 418,6 wolte ich würken wariu werc sunder oder âne allen willen. - 418, 20 als ob nieman si noch nieman lebe 
noch uf daz ertriche nie kein mensche komen si. - 418, 24 ein armuot an dem hoesten und an dem groesten.

Vom tractat VI (s. 448-475) habe ich durchgesehen die seiten 448-452. 457. 462. 467. Von diesen enthalten die seiten 449. 452. 457. 462. 467 keine belege, im rest stehen $1+3+$ 2 beispiele:

448, 26 gelobet unde geeret si der name. - 450, 10 der vierde gebreste ist nit unde haz, wa der ie nit unde haz gewunne. - 450,17 solt in also lange an schrien unde weinen. - 451,9 so sulle wir der sünden vergezzen und alle die gebresten unsers ebenkristen. - 451, 15 der gote neher ist wan du unde glicher.

\section{Der tractat VIII (s. 478-483) enthält folgende belege:}

478, 27 fride unde friheit des herzen in einer stillen ruowe. - 479, 3 sin selbes uz gan unde verlouken. - 479,9 ein wurzel aller tugende unde guotheit. - 479, 23 da entdecket und enbloezet got der sele sin geburt, - 479, 31 da sich got mit minne und mit pfliht verstricket unde gelobet hat zuo der sele. - 479, 39 ein not einer gewalt oder einer notsache. 480, 3 sint sie ze kleine unde ze snoede. - 480, 40 tugent unt elliu guotiu werc. - 481, 1 als andaht und innekeit. - 481,5 dringet oder trücket den geist. - 481, 24 gesetzet sin in fride und in ruowe. - 481, $31 \mathrm{daz}$ diu ewege regele unde diu ewige gesetzede an uns vollebraht werden. 481, 38 welchez die gaben oder die frühte sin, die den menschen gegeben werden. - 482, 14 daz den menschen ungelücke unde liden niht betrüeben müge. - 482, 15 noch gelücke und ere der welt niht erfroüwen müge. 482, 30 lockender rede unde zuojehender rede. - 483, 1 swer dirre stücke niht an im hat unde dar an versumet ist.

\section{Tractat IX (s. 483-4.93) bietet folgende belege:}

483, 34 habe mit erneste unde mit ganzem vlize gesuochet. - 483, 34 welhiu diu beste unde diu hoehste tugent si. - 484, 4 alse verre min vernunft orziugen unde bekennen mag. - 484, 8 wer unbetriebet unde luter wil sin. - 484, 17 got kan sich einfüeglicher füegen zuo mir unde baz vereinen mit mir. - 484,34 der ist also einvaltic unde kleinvüege. 484, 36 ein ieclich enpfenclich dinc wirt empfangen unde gevazzet in sime enpfahende. - 484, 38 ein ieglich merklich dinc wirt gemerket unde verstanden. - 485, 17 vollekomeniu abgescheidenheit enhat kein ufsehen noch keine neigunge under keine kreature noch über keine creature. - 486,36 gegen allen zuovellen liebes unde leides, eren, schande unde lasters. 487, 8 diu gnade ziuhet den menschen von allen zitlichen dingen unde liutert in vou allen zergenclichen dingen. $\rightarrow$ 487, 19 wirt got niemer deste milter noch deste geneigeter gegen den menschen. - 487,19 mich wol merken unde rehte verstan. - 487, 33 welchez gebet und andaht er hoeren solte. - 487, 34 mit flize anruofen unde mit ernste biten. - 487, 34 unde daz anruofen unde gebet wil got niht morgen erhoeren. - 487, 36 ist aber din gebet niht redelich und ane ernest. - 488,4 helt diu dinc nach dem

Beiträge zur geschichte der deutschen sprache. $\mathrm{XXXlV}$. 
loufe unde nach der ordenunge, die er in hat gegeben. - 488, 39 ein wises uud ein leiter ist. - 489,6 heizet der mensche sinnelos unde verzucket. - 489, 12 die alle ir sinne nnde gedanken kerent uf zergenclichiu güeter. - 489, 18 daz der uzer mensche mac in üebunge sin, daz doch der inner mensche des genzliche ledic stet und unbewegélich. - 490,11 als er bereitschaft und enpfenclicheit vindet. - 491, 35 wer nu vollekomener abgescheidenheit adel und nutzen merken wil. - 492,6 kein liplicher und fleischlicher lust. - 492, 35 daz zuoval, anhaftunge und kumber bringen mac. - 493, 5 wa nach du stellen unde begerunge haben solt. $-493,9$ sol din meinunge unde din fluht dar inne sin.

Das sind $2+6+1+1+7+2+3+1+1+2+2$ beispiele.

Der kurze tractat X (s. 493, 14-495, 25) bietet nur $2+$ 2 belege:

493, 20 in ir selber gezeiget unde geordent hat: - 493, 32 niht ergrifen noch ergründen mügen. $\div 494,7 \mathrm{der}$ minnet werliche unde sicherliche. - 494, 33 allez, daz si hat ze lazen unde ze geben.

Von tractat XI (s. 495-516) habe ich die seiten 496, 500, 504, 508, 511, 513 geprüft; die seiten 496, 504, 508 bieten 2 $+1+4$ belege, die übrigen gar keine:

496, 17 mit dem, daz èz verstet unde daz ez begriffen hat. - 496, 23 dringet und drücket sich in den willen. - 504, 9 scheidet die sele von allen creaturen und bewegelichen dingen. - 508, 28 si enmüieze e engan allen bilden unde formen. - 508, 30 si muoz ouch abe gan und entsinken irre eigen bewegelicheit. - 508, 40 lidic unde bloz sin alles gemerkes unde prïevens und aller sinnelicheit.

Der tractat XII (s. 516-520), der auch durch seinen wortschatz eine eigentümliche stellung einnimmt, enthält überhaupt nur 2 beispiele:

520,20 daz nebeste unde daz subtileste wort, daz creature geleisten mac. - 520, 26 wone in dem obersten mit gedenken und mit minne.

Der tractat XIII (s. 521-527) bietet im ganzen 6 belege:

521,10 swebet unde wonet in einer steten unberüerlichen stilheit. 522,2 uiber unser herze unde verstentnisse erhaben werde. $-523,6$ blibe ir da kein bekennen oder versten des nihtes. - 523, 8 allez bekennen unde vernemen des nihtes. - 523,16 allez bekennen und vernemen. - 526,9 swie er wil, swanne er wil und als er wil.

Der tractat XIV (s. 527-533) enthält 2 belege:

530,15 rou nieman geborn noch gevlożzen. - 532, 30 si ist als arm und als bloz und als ledic.

Der tractat XV (s. 533-542) enthält 3 belege:

534, 12 ein geschepfunge sines vaters personlich unde wesenlich. - 
538,18 got ist ein einveltic guot an ime selben und ungestücket. $-538,24$ den verbrunnen geisten unde die in dem brande erloschen sint.

Der tractat XVI (s. 542, 9 - 543, 13) enthält 2 belege:

542,10 ist rot unde zornic. - 542,23 so wolte ich, daz er mich verdilgete und wolte e ze nihte werden.

Der tractat XVIII (s. 578-593) enthält auf den seiten mit ungerader zahl folgende belege:

579,4 ob allen worten und ob allen namen swebet. - 585, 5 da alliu dinc bluz unde ledic wider in gefüeret werdent. - 581, 10 sit ich was blibende und ruowende uf eigenschaft der nature. - 581,16 do wart ich blibende unde ruowende uf gotes nature. - 581,19 ich stuont bloz unde ledig ungeschaffen. - 581,32 da wirt ir genomen kraft unde leben. 583,14 sin wesen unde sin ufenthalt. - 583;15 verstande mit einer frien ledigen vernunft. - 583, 36 in blozer lediger müezikeit. - 583,38 muoz ich würken mit einer frier lediger müezikeit oder unbewegelicher stillekeit. - 583, 39 von mir selber unde von miner selbheit unde von allen dingen, diu zit unde zitlicbkeit besitzen mac. - 585,1 daz diu sele widerbildet und überbildet. - 585, $13 \mathrm{daz}$ er si in einem müezigen oder stillen geiste. - 585, 35 ein ufenthalt geistlicher forme unde vernünftiger bilde. $-587,14$ da ist menschlichiu nature bewegelich unde nimt zuo und abe. - 589, 31 diu selbe welt ist ein vinstri und enhat des liehtes nie bekant. - 591,24 sol bloz sten unbedecket. - 593, 29 hant dise menschen glorie und ere. 593, 31 das sie werliche got besitzent und in sich besliezent.

Das sind $1+5+7+3+1+1+2$ beispiele.

Das ergebnis unserer betrachtungen geht dahin, dass die tractate I-IV, VI, VIII-XVI und XVIII nicht vom verfasser des tractats $V$, also nicht von meister Eckhart herrühren können.

Dieses ergebnis bestätigt zu einem teil zweifel an Eckharts urheberschaft, die bisher schon ausgesprochen worden sind.

Dass tractát III von Eckhart stamme, hat man insbesondere wegen 414, 37, 38 geleugnet, vgl. Deutsch, Haucks realencyclopädie ${ }^{3} 5,147,31$. Auch tractat VI ist bereits als 'pseudoeckehartisch' verdächtig, vgl. Haupt, Haucks realencyclopädie ${ }^{3}$ 3, 470,36; gegenüber den bedenken, die Denifle an verschiedenen stellen ausgesprochen hat, bemerkt freilich Otto Simon mit recht: 'den nachweis für die richtigkeit seiner behauptung ist er uns freilich schuldig geblieben', überlieferung des tractates'Schwester Katrei', Hall. diss. 1906, s. 3.

Den tractat XVIII hat widerum schon Denifle. Eckhart abgesprochen, Buch von geistlicher armut s. II, weil in der 
(von Pfeiffer willkürlich geänderten) überlieferung von Eckhart in der dritten person gesprochen wird; ein vielleicht nicht durchaus zwingender grund; die ähnliche formel in predigt $X V$ ist bei Wackernagel, Altdeutsche predigten s. 405 als zeugnis für die aufzeichnung durch dritte hand gefasst. Was Denifle dann Archiv f. literatur- und kirchengeschichte II 676 beibringt, lässt sich auch kaum als ein beweis bezeichnen.

Eine mittelstellung zwischen dem tractat $V$ einerseits und der masse der übrigen anderseits nimmt tractat XVII ein (s. 543 -578). Ich verzeichne die beispiele der widerholung für die seiten 544-550:

544, 24 sin erster hoehster fliz. - 544, 26 daz kreftigeste gebet unde vil nah daz allermehtigeste alliu dinc ze erwerbenne. - 544,30 ie daz gebet unde werc kreftiger, wirdiger, neher, lobelicher unde vollekomener ist. - 544, $32 \mathrm{daz}$ mit nihte beladen ist oder beworren ist. - $544,36 \mathrm{ez} \cdot$ neme hie inne sine kraft unde sin vermügen. - 545,5 daz mir also wol mit gote were und also vil andaht hete und fride mit gote. - 545, 21 da vindest du hindernisse und unfride. - 546,1 als si sin fri eigen weren unde sie besezzen hete mit ganzem gewalte. - 546,14 ez ist reht ein geliche widergeit und ein gelicher kouf. - 546,17 da heb an unde des la dich kosten allez, daz du geleisten maht. - 546, 26 als verre wir sin und wesen han. - 546, $33 \mathrm{daz}$ des menschen wesen unde grunt guot si. 546, 37 aller din ernst unde fliz. - 547, 11 allez, daz ungelich uude fremde ist. - 547, 14 zügen sich sere von den liuten unde weren gerne alleine. 547,27 des ist daz werc eigenlicher unde werlicher. - 547, 32 er enmeinet niht noch ensuochet niht noch ensmecket ime niht dan 'got. - 547, 35 enmac disen menschen niht zerstrewen noch vermanicveltigen. - 548, 1 got habe in gegenwürtekeit in dem gemüete und in der meinunge. - 548, 9 ein gelichez gemüete haben und ein gelich getruwen. - 548,16 minnet worte meinet in niht alleine. - 548,22 so were im in allen steten gar. rehte unde wol. - 548, 26 an eime zuokeren unde meinen gotes. - 548, 28 daz were sere swere und ouch daz allerbeste niht. - 548, 29 der mensche sol niht haben noch im lazen genüəgen. - 549,4 als vil mer und inwendiger unde gegenwärtiger unde steter ist daz bilde des trankes. - 549,6 daz im niht anders smecket unde ze herzen gat. - 549, 8 wes er beginnet oder waz er tuot. - 549, 9 in allen dingen vindet er des dinges bilde und ist ime also gegenwärtic. - 549,14 hie zuo gehoeret fliz unde minne ... und ein wacker war vernünftic würklich wizzen. - 549,18 daz er diu dinc fliuhet unde sich an die einoede keret. - 549,19 wer oder bi wem er ist. - 549, 23 er muoz sich vil unt dicke üeben. - 549,24 wie sur unde swer ez ime doch werde. - 549, 25 wil er ez flizeclichen üeben unde dicke. - 549, 29 schribet er ledeclichen unde frilichen. - 549, 38 mit götlicher gegenwärtikeit durchgangen sin und mit der forme sins geminten gotes durchformet sin. - 550,6 niht stat gehaben noch vinden. - 550,11 
er milge bier an ane underlaz wahsen unde me gewinnen in einem waren zuonemen. - 550, 22 dar zuo muoz fliz gehoeren unde muoz kosten allez daz man geleisten mac. - 550,33 siner vernunft gewaltig ist unde der gebruchende ist.

Das sind $5+2+6+5+7+13+4$ belege. Ich zähle weiter an beispielen: 551: 6, 552: 6, 553: 12, 554: 7, 559: 11, 558: 4, 569: 16. Das geht über die zahlen für den unbewussten durchschnitt beträchtlich hinaus, aber es bleibt zurück hinter dem tatbestand in tractat $V$. Dort kommen im mittel 13 beispiele auf die seite, hier nur 8. Für den fall, dass jemand bedenken tragen sollte, den tractat XVII auf grund dieses abweichenden zahlenverhältnisses dem verfasser von tractat $V$ abzusprechen, sind wir glücklicherweise in der lage, noch andere tatsachen zur entscheidung heranzuziehen.

Der verfasser von XVII liebt es, die erörterung dadurch weiterzuführen, dass er selber fragen stellt oder von andern stellen lässt:

544, $6 \mathrm{nu}$ merke, waz wil er mir? Daz ich niht wil. - 544,31 daz ledige gemüete vermag alliu dinc. Waz ist ein ledic gemüete? Daz ist ein ledic gemüete, daz ... - 547, 24 den menschen enmac nieman gehindern. War umbe? Da hat er alleine got. - Aehnlich 545, 28. 548, 24. 352,31 . 354,32 . 562,33 . 36. 563,16 . - 570, 12 ein frage: war umbe was si niht bereit? Ebenso 571, 20. 574, 16. - 573, $11 \mathrm{nu}$ ist ein frage: wie möhte ich dise minne han? Ebenso 574, 5.

Oder der verfasser lässt sich die frage von aussen heranbringen:

547,14 ich wart gefraget, obe daz daz beste were. - 572, 39 da wirt gefraget, war umbe denne got niht neme die linte. - 553, $6 \mathrm{nu}$ möhtest du fragen, wa der wille ein reht wille si. - 562, 3 so möhtest dn sprechen: wes habent denne unser vorvaren getan? Aehnlich 566, 9. 39. 566, 7 .

In tractat $\mathrm{V}$ findet sich $\mathrm{zu}$ diesen 22 beispielen ein einziges sicheres seitenstück:

423, 37 wie sol der tuon? wie tuont arme liute?

Eine zweite stelle steht der rhetorischen frage sehr nahe:

426, $33 \mathrm{nu}$ merke: waz wunders unde waz lebennes hat der mensche uf der erden als in dem himele in gote selben! ime dienet ungemach in gemach, und leit gelich als liep.

Aber nicht nur fragen legt der tractat XVII den lesern in den mund, sondern auch erwägungen und bedenken anderer art: 
545,5 die menschen sprechent 'eya, herre, ich wolte gerne, daz mir also wol mit gote were.' - 551,1 vil liute sprechent: 'wir haben guoten willen'; sie habent aber niht gotes willen. - 562, 24 nu möhtest dú sprechen: 'unser herre Jesus Kristus der hete ie die hoehste wise, dem süllen wir iemer von rehte nachvolgen'. - 565, $29^{\prime}$ nu möhtest du sprechen : 'eya, herre, ich vinde mich alse bloz unde kalt unde trege, dar umbe tar ich niht zuo unserm herren gen'. Aehnlich 566, 9. 569, 3. 576, 19. 577, 27. - 566, 21 'eya, herre, ich wolte in geine loben, so enkan ich niht'. Ebenso $566,18$.

Nichts derart in tractat $\mathrm{V}$.

Ein sehr beliebtes äusseres mittel zur weiterführung des gedankens ist in tractat XVII die conjunction mêr:

545,17 daz ist niht schult, daz dich die wise oder diu dinc hindernt, mer: du bist eż selber. $-545,27$ sie gant als einer, der eines weges vèrmisset: ie verrer er gat, ie mer er irret. Mer: waz sol er tuon. - Vgl. ferner 545, 37. 546, 26. 548, 20. 31. 40. 549, 35. 550, 6. 30. 554, 31. 39. 555, 16. 559,24 . 560,1 . 563,18 . 564, 7. 567, 17. 20. 568, 7. 569, 31. 574, 2. 3. 575, 8 . $576,16.34$.

Diesen 26 beispielen stehen in tractat $V$ nur 3 gegenüber: 419, 26. 430, 31. 431, 17.

Auf dem gebiete des wortschatzes lässt sich die wahrnehmung machen, dass XVII mit fremdwörtern und ihren ableitungen erheblich freigebiger ist als $V$. So stehen in XVII und fehlen in V: forme (XVII 549, 39) - durchformet $(549,39)$, glorie $(573,12)$, jubilus $(553,35)$, $\operatorname{llar}(556,13)$, penitencie $(560,7$. 15. 21. 23. 29. 34), punkt $(570,19)$, sacrament $(565,19$. 25. 33. $566,27)$, - sacramentlich $(568,7.17 .24)$; dem hat tractat $\vec{V}$ von ungefähr gleichartigem nur glose $(437,4$, in XVII fehlend) gegenüberzustellen.. Und XVII bezitzt contemplieren $(543,26)$, jubilieren $(553,23)$, studieren $(546,6)$, die in $V$ nicht nur selber, sondern auch ihrer art nach unvertreten sind. Es liegt nicht ferne zu vermuten, dass zwischen der lehrhaften deutlichkeit, die sich in der starken verwendung der widerholung durch tractat $V$ ausspricht, und der enthaltsamkeit in bezug auf fremdwörter ein innerer zusammenhang besteht.

Von grosser bedeutung sind endlich die versicherungspartikeln, die auf beiden seiten angewant werden. Tractat $V$ bestreitet seinen bedarf hauptsächlich mit sicherlichen und werlichen (gewerlichen); - sicherlichen steht 421,15. 422,1. 7. 423, 23. 427, 4. 15. 37. 428, 3. 31. 34. 429, 30. 440,2. 441, 9. 442, 12. 443, 20. 
441,31. 446, 17; werlichen findet sich 421, 17. 422, 12. 15. 30. 36. 427, 40. 428, 20. 429, 11. 32. 436, 47. 438, 5. 38. 440,10. 441, 4 . 442, 5, 24 (gewerlichen). 38. 443, 33. 446, 7. 28. 447, 13; dreimal steht in der warheit: 428,21 . 431, 32. 440,3. In tractat XVII dagegen herscht in der vvarheit: $544,9.545,10.19 .29 .547,1$. 3. 17. 20 (zweimal). 29. 548, 12. 550, 26. 27. 551, 34. 552, 32. 36. 553,2 . 4. 6. 554, 6. 10. 555, 15. 32. 38. 40. 556, 5. 30. 36. 558, 34 . (564, 19 in der überschrift). 567, 3. 572, 16. 574,30. 40. 575, 21. $576,31.39$. 577, 6. 17. 22 , also nicht weniger als 40 mal. Daneben ist triuwen, entriuwen öfter belegt: 548, 10. 549, 7.23. $550,18.31$. 553, 23. 566, 11. 567, 17. 570, 17. 574, 35; ganz vereinzelt werliche 576, 5, gewerliche 569, 30. 570, 34 (werliche 546, 1. 548,25 gehört nicht hierher).

Danach kann wol kein zweifel mehr bestehen, dass tractat $V$ und tractat XVII, in denen man bis jetzt die festesten grundlagen für die erkenntnis Eckharts gesehen hat, von verschiedenen verfassern herrühren (vgl. noch s. 551, NS.).

$\mathrm{Zu}$ keiner bestimmten entscheidung ist wegen seines geringen umfangs bei tractat VII (s. 475-478) zu gelangen. Die versicherungspartikeln fehlen durchaus, aber das kann bei einem umfang von wenig mehr als zwei seiten lediglich zufall sein. Von der widerholung finden sich folgende beispiele:

475, 35 ez koment vil liute ze klarem verstantnisse und ze vernünftigem underscheide bilde unde forme. - 476,7 koment über verstantlichez schouwen und über vernünftige begrifunge bilde unde forme. - 476, 7 gerïcket unde gebüret über vernünftige begrifunge. $-476,10$ in dem vindet got sine ruowe unde sinen fride. - 476,11 ze wonende unde ze würkende, wie er wil. - 476,14 ir leben ist verborgen und ir wesen allen den, die ... - 476, 17 mit luterr verstantnüsse unte mit klarer vernunft. - 476, 20 an sinem wesen und an sinem leben. - 476, $21 \mathrm{daz}$ der geirret hat und ewecliche vervallen ist von der ewigen. warheit. - 476, 25 daz ez nütze und ein notdurft si, daz man flizic war nemen unde bekennen unde brüeven sol die invelle des liehtes unde die klarheit der vernunft unde des schouwens. - 476,29 welt ir nu wizzen unde bekennen die gerehten vernünftigen gewereu anschouwer gotes. - 476, 30 die da niht betriegen noch gevelschen mac. - 476, 33 der aller hoehste meister an kunst und an wisheit und an hoher vernunft. - 476,39 ir werdet min jụnger an kunst und an wisheit und an hoher vernunft. - 477, $16 \mathrm{daz}$ er sich mit minne unde mit triuwen zuo dem niht behalten hat. - 477,7 daz sie sint ledic worden ir selbes unde ledigent sich $\mathrm{uz}$ in selber. -- 477,10 sie habent sich gote lazen ganz und gar. - 477, 17 sie fiiegent unde verbindent sich also nahen zuo gote. - 477, 20 sie nietent sich nnde gebruchent gotes. - 477, 25 von deheinem 
geluste noch smacke der nature noch der creature. - 477, 30 sie sint gewafent unde gezieret mit allen tugenden. - 477,34 sie sint vollekomen unde gerecht.

Also auf s. 47619 beispiele, auf 477 deren 9 .

Das stimmt zum gebrauch von tractat $V$, aber auch in tractat XVII begegnen zahlen von ähnlicher höhe. Bemerkenswert ist, dass in VII die fremdwörter forme $(475,36.476,2)$, formelich $(476,3)$, klâr $(475,35.476,18.477,4)$, klarheit $(476,27)$, punct $(476,10)$ auftreten; die dem tractat $V$ dnrchaus fremd sind, während sie dem gebrauch von XVII nicht widersprechen würden, vgl. s. 542 .

Aus der masse der Eckhart abzusprechenden tractate hebt sich noch tractat VI heraus. Er ist gegenüber den andern gekennzeichnet durch die starke neigung, dem leser seine weisheit mit wizzest, wizzet, du solt, ir sult wizzen ans herz zu legen: 450, 20 wizzest für war, daz muoz sin, 450, 22 vizzist, daz nidigiu wort alse schedlich sind. Ebenso 450, 28. 32. 451, 5. 7. 31. 453, 1. 34. 454, 12. 456, 13. 457, 1. 7. 31. 458, 1. 3. 26. 29. 32. 462, 24. 29. 35. 464, 4. 32. 36. 468, 40; wizzet: 453,4 got hat uns für gesehen zuo siner ewigen selikeit; ebenso 453, 39 . 454, 26. 455, 3. 6. 20. 24. 456, 36. 457, 2. 459, 12. 460, 28. 463, 11. 29. 464, 17. 465, 10. 468,4. 469,8 (zweimal). 470,5. 24. 471, 34. $472,1.473,21.27 .470,15.20$. 24. 475, 17; du solt wizzen 462, 29. 464, 35. 472, 27. 474,13; ir sult wizzen 462, 12. 23. 468, 1. 3. 469, 6. 27. 35. 471, 33. 35. 472, 4. 474, 3. 12.19.

Was nach ausscheidung der von drei verschiedenen verfassern herrührenden tractate V, VI und XVII übrig bleibt, ist natürlich noch immer keine einheitliche masse. Ich weise darauf hin, dass in XI, XII, XIV, XV die zahl der widerholungen nur eine ganz verschwindende ist. Unter diesen nimmt wider XII eine sonderstellung ein. Einmal durch die verhältnismässig grosse zahl von wörtern (auf $4 \frac{1}{2} 2$ seiten!), die nur hier belegt zu sein scheinen: begrifenlicheit, durchgrundelicheit, durftlos, entgeistikeit, geursprungetheit, gevoordenheit (420, 15, fehlt bei Lexer), ingegeistikeit, ungewordenheit, daz unbegrifen, unverdruclich, uzgeursprungetheit, widerbloeze. $Z$ weitens durch die eingestreuten reime, die Pfeiffer nur zum kleinen teil bemerkt hat; ich verweise auf 516,14 himelrich : dich. - 516, 16 stat : hat. - 516,28 gezogen : betrogen. - 
516,32 hô : alsô. - 516,34 apgrunt : kunt. - 517,6 nihte : ihte. - 517, 12 wortelos : bloz. Dabei habe ich die zahlreichen schlüsse auf -heit noch unberücksichtigt gelassen.

Es wäre nun die frage, ob mit den von uns gewonnenen kennzeichen noch über die tractate hinaus etwas auszurichten ist.

Auf eine prüfung der splitter, die im Liber positionum vereinigt sind, verzichte ich von vornherein. Aber auch die ausdehnung der untersuchung auf die predigten, die unter Eckharts namen gehen, stösst auf sehr erhebliche bedenken.

Erstens besitzen wir keine vollgiltige kritische ausgabe der in betracht kommenden texte. Pfeiffer bietet kein bild der weitverzweigten überlieferung und hat sich mehrfach eigenmächtigkeiten erlaubt (einen besonders schweren fall hebt Denifle hervor: das buch von geistlicher armut s. II).

Zweitens würden schon die grundlagen unserer überlieferung sehr unzuverlässig sein, wenn die landläufige anschauung recht hat, nach der wir im wesentlichen aufzeichnungen des gesprochenen worts nicht vom prediger selbst, sondern von anderer hand besässen; vgl. Wackernagel, Altdeutsche predigten s. 405; Linsenmayr, Geschichte der predigt in Deutschland s. 395; Jostes, Collectanea Friburgensia IV; s. Ix; Pahncke, Untersuchungen zu den deutschen predigten meister Eckharts s. 23; Steinmeyer, Haucks realencyclopädie ${ }^{3}$ 2, 651, 7; Deutsch, ebda. 5, 147, 37; für 'die predigten Bertholds, Geilers und wahrscheinlich auch diejenigen Taulers' wird es auch von F. Landmann angenommen, Das predig:twesen in Westfalen s. 104, anm. 4. $\mathrm{Ob}$ das in der tat für einen grossen teil der texte zutrifft, ist zweifelhaft; jedenfalls liegen bis jetzt keine genügenden beweise für diese anschauung vor. 1) Was man als

1) Mein college Schian macht mich auf folgende stelle aus dem 15.jh. aufmerksam: 'dise bredigen hat angenese steffan sahssen dohter gehoert bredigen und het sie behalten in irem hertzen und hett sü geschrieben und det sï abe ir geschrift anderwerbe schriben', bei Pfleger, Zur geschichte des predigtwesens in Strassburg vor Geiler von Kaysersberg s. 22. - Ich verweise ferner anf eine stelle aus dem angeblichen leben Taulers, das ich nach Arnolds Kirchen- und ketzerhistorie, 1742, III 664 citiere. Es heisst da s. 664: 'so balden die predigt geendigt war, verfügte sich dieser mann iu seine herberge, und schrieb den gantzen sermon, von wort zu wort, recht 
hauptstütze für sie betrachtet, die 'zerlesung' der predigten, das mosaikartige vieler stücke, das kann bereits für den urtext der predigten kennzeichnend gewesen sein; vgl. die ausführlichen darlegungen in der eingangs erwähnten untersuchung Spämers über zersetzung und vererbung in den mystiker-predigten. Nehmen wir aber einmal an, dass unsere predigttexte hauptsächlich durch solche nachschriften auf uns gekommen wären. Dann wären gerade die eigentümlichkeiten leicht der zerstörung ausgesetzt gèwesen, die bei unserer kritik der tractate eine wesentliche rolle gespielt haben, die widerholung und die.verschiedenen kleinen hilfsmittel, die die rede für den hörer eindringlicher gestalten sollen.

Drittens wäre es wol möglich, dass in der predigt von vornherein andere stilmittel angewant würden, als im tractat. $Z$. b. könnte ein mann, der in der ruhigen darlegung des tractats die weiterführenden fragen vermeidet, sehr wol in der predigt von ihnen gebrauch gemacht haben.

Endlich viertens ist der umfang der meisten predigten recht gering im vergleich $\mathrm{zu}$ dem der tractate, so dașs das fehlen einer erscheinung in einer predigt vielleicht lediglich folge des zufalls ist.

Trotz dieser bedenken möchte ich unter allem vorbehalt wenigstens einige wahrnehmungen mitteilen, die ich ge= macht habe.

Zunächst stelle ich fest, dass unter den predigten, die Pfeiffer, Sievers (Zs. fda. 15, 373), Jostes (Collectanea Friburgensia IV), Jundt (Histoire du panthéisme au moyen âge s. 231), Pahncke (Zs. fda. 49, 395), Wackernagel (Altdeutsche predigten s. 156) als eckhartisch veröffentlicht haben, keine

wie ihn der Doctor ausgesprochen hatte, auf, gienge darauf zu dem Doctor hin und sprach: Ich habe euren sermon aufgesehrieben, und dafern es euch nicht verdrüszlich seyn würde, wolte ich ihn herlesen. Der Doctor sagte: ich höre es gern. Und der mann lase den sermon, und sprach zu dem Doctor: Lieber Herr, ich bitte euch, durch Gott, dasz ihr ihr mir saget, ob mir auch ein wort fehle oder mangle, damit ich solches hinzufüge. Da antwortete der Doctor: Lieber Sohn, du hast es so recht geschrieben, nach aller weise und worten, als es aus meinem munde gegangen ist; und wisse, dass wenn mir einer gleich viel gelt geben wollte, könte ich es ihm nicht so eigentlich zu worte bringen, als du es hier geschrieben hast, ich hätte mich denn anderwärts in der schrift darnach bearbeitet. 
den starken procentsatz von widerholungen aufweist, wie der tractat V. Von den versicherungspartikeln kehrt sicherliche, das in tractat $V$ eine so grosse rolle spielt, nur in zwei predigten wider: Pfeiffer III (s. 16-26) und IV (s. 24-30). Daneben steht aber in diesen beiden texten nicht nur werliche, gewerliche, sondern auch in der warheit, das im tractat $\mathrm{V}$ mit seinen 30 seiten im ganzen dreimal vorkommt, und auch entriuwen, das in tractat $\mathrm{V}$ gänzlich fehlt. Folgendes sind die belege: sicherliche 16,24. 18, 40. 26, 2. 26, 22. 27, 29. 28, 29. werliche 25,16 . 26,$40 ;$ gcwerliche $25,2.25,17$. - in der warheit 17, 27. 24, 31. 25, 9. 25,32. 27,15. 28,32. 28,33. - entriuwen 20, 8. 26, 5. Dann kommt aber noch ein anderer unterschied gegenüber tractat $V$. In den predigten III und IV begegnen mehrfach die fremdwörter forme 17, 32. 21, 10 (förmelich 21,36). - materie 19, 23. 21, 8. 21, 9. 28, 32. - penitencie 29,12. $29,14$. 29, 26. - punt 20,4. 20,8. 27,32. 27, 37. 28,6. Keines von diesen steht in tractat $V$, das, wie wir s. 542 sahen, in sachen der fremdwörter grosse zurückhaltung übt.

Haben wir somit keinen bestimmten anlass gefunden, eine der predigten für Eckhart in anspruch zu nehmen, so wird man zweifeln müssen, ob wir überhaupt eine erhebliche anzahl von Eckharts predigten besitzen; es wäre doch sonst ein höchst merkwürdiger zufall, wenn die art der überlieferung die für ihn so bezeichnenden eigentümlichkeiten überall verwischt hätte.

In den predigten bei Pfeiffer, I, XI und XC, findet sich trinwen, in triuwen neben in der warheit verwendet (triuwen 6, 6. 58, 26. - in triuven 299,3 . - in der warheit $3,22.10,14$. $58,13.59,27$. 59,38 . 297,9 . 298,13); also die partikeln, die den tractat XVII kennzeichnen. $\mathrm{Zu}$ dessen weise stimmt auch die zahl der widerholungen:

3,9 in uns geschehe oder vollebraht werde. - 3,14 der ist zemale verre und unwizzende von dirre gebürte. - 3,21 in dem aller lutersten und edelsten und subtilsten. - 3,26 sich gar luter haben unde gar adelliche leben unde gar einic unde gar inne. - 4,2 inne sin und einic sin. $-4,6$ da mit er erwerbe unde verdiene, daz disiu geburt in ime geschehe unde geborn werde. - 4,8 in siner vernunft und in sinen gedanken. 4, $19 \mathrm{ez}$ get iu mer in unde baz. - 4,34 anders enmac si kein gesehen wirken noch geben. - 4, 40 ruowe und ein wonunge zuo dirre gebürte nude zuo disem werke. - 5,9 so nement sie unde schöpfent bilde unde gelichnisse. $-5,21$ kein bilde geschöpfen mac noch geziehen. $-5,26$ ist 
fri und ledic. $-5,28$ ane bilde oder gelichnisse. $-5,34$ in ir meisterschaft und in irm werke. $-6,2$ ane allez mittel, bilde oder gelichnisse. $-6,6$ in bilden unde in gelichnisse. - 6,24 sin unde wonen in dem wesen und in dem grunde. $-6,26 \mathrm{daz}$ enmeinet noch enwiset sich selber niht. $-6,27 \mathrm{ez}$ ziuhet unde wiset. - 6,33 da mit er erwürbe únde verdiente, daz disiu geburt in ime geschehe unde vollebraht werde. $-6,35$ ein inbilden und ingedenken an got. - 6,36 in eime swigen oder in einer stille und in einer ruowe. - 7,4 daz allerbeste ist und alleredelst ist. - 7,18 daz si den licham füeren unde zieren solden. - 7,19 der brant unde diu hitze. - 7,29 in fride und in ruowe. - 7,31 kein bilde oder gelichnisse. 7, 32 warheit unde wesen. - 8,7 in dem grunde und in dem wesen. 8,9 sie elliu dinc müezent nemen unde bekennen in ir eigem bilde. $-8,27$ in eime geriune und in einer stille. $-8,28$ muoz man und sol ime nach lonfen. - 8,30 jamern unde siufzen. - 9,7 daz vorspilt unde vorblicket miner sele. $-9,9$ vollebraht unde besteteget in mir. $-9,12$ benemen unde verstelen. - 9,13 bewiset und offenbaret. - 9,14 berouben unde benemen. $-9,17$ versmalz unde zerfloz. $-9,29$ muoste in vil verborgen sin unde bleip in unbekant. - 9,37 den nutz unde die fruht dises heimelichen wortes unde diss vinsternüsses.

Also $4+8+6+10+6+5+9$ beispiele: durchschnitt nahezu 7.

58,4 bitterkeit und schaden und übel und ungemach. - 58,6 nngemach unde bitterkeit. - 58,12 umbe dinen eigenen nutz oder umbe din eigen selekeit. - 58,39 ein gewissiu warheit und ein notwarheit und ein geschriben warheit. - 59, 1 er möhte dir ez niht verbergen uud er müese dir ez offenbaren. - 59,7 enhat niht ze schaffene mit der zit und enweiz niht von der zit. - 59,20 si wil etwaz edelrs, etwaz bezzers. - 59, 25 als er ein wurzel ist, eịn ader, in der urspringet güeti.

Also $4+4$ beispiele.

$296,17 \mathrm{daz}$ si vil wil begrifen unde han. - 296,19 so enkan got niemer gewonen noch gewürken in dir. - 296, 21 in einer hoehern unde bezzeren wise. - 296, 28 so enwere mir nihtes niht swere noch enwürde niht alse schiere betrüebet. - 296, 31 allin swere, leit und erbeit. 296, 31 zit unde zitlich dinc han ïbertreten. - 296, 32 sin wir fri unde fro. - 296. 38 sin uzfliezen noch sin uzblüegen. - 297, 4 machet ez warm unde heiz. - 297, 6 stiller unde geruowiger. - 297, 14 swenne ander dinc sint uz unde vol. - 297, 24 so hoch unde so edel. - 297, 38 bloz und unbedecket. - 298, 7 wir sülen ez gote endecken unde geben. $-298,19$ sie meinent vil und wellent als vil. - 298, 21 suochet oder meinet. - 22. 23. - 298, 24 deme entecket got unde git ime. - 298, 29 diu zunge hat eine tecke und ein kleit. - 298, 32 mit dem kleide unde dem mitel. - 298, 35 swere unde bitter. - 298, 39 in süezikeit und in gemache. - 298, 40 in liden, in ungemach und in erbeit.

Also $8+5+11$ beispiele: durchschnitt stark 7. Im tractat XVII waren es im mittel 8 beispiele auf die seite. 
Von den predigten, die das imperativische wisst aufweisen, können natürlich nur solche zu tractat VI in beziehung gesetzt werden, bei denen die formel häufiger auftritt, das sind höchstens die predigten bei Pfeiffer XXXVII. XL. XLII. LXXX und Jundt no. 6. - Folgendes sind die belege für die formel: XXXVII: wizzent 127, 39. 40. 128, 11. 15. 20. 28. 31. 129, 2. ir sunt wizzen 127, 38. 128, 40. XL: wizzest 136, 14. 15. 137, 24. - wizzent 134,22. - ir sunt wizzen 134,8. XLII: wizzent 141, 33. 143, 19. - ir sullent wizzen 141, 11. LXXX: wizzent 256, 23. 256, 30. 257, 21. 22. 27. 258, 28. Jundt no. 6: wizzent 254 viermal, 255 zweimal.

Von diesen zeigt jedoch nur XXXVII noch weitere eigentümlichkeiten, die auch im tractat VI vorliegen. So die häufige verwendung der mit verstan gebildeten formeln: daz sulen wir also verstan 126, 8. 9. 12. 127, 16. - verstant 127, 12. - ir sunt verstan 127, 23. 128, 26. 129, 1. Vgl. in tractat VI: sullen wir verstan 448, 30. 452, 34. 453, 33. 454, 34. 35. 459, 24 . 460, 15. 462, 20. 466, 26. 473,13. - un verstant 471, 14. - soltu verstan 456, 11. 469, 17. - sult ir verstan 455, 18. 455, 35. 460, 13. 469, 40. 470, 15. 470, 27. 39. 471, 11. 37. 472, 10. 22. 29. 474, 29. 34. - Ferner die wendung: ich spriche mê 128, 16. 16; vgl. in tractat VI: 454,15 . 17. 20. 456,35. 458, 39. 459,6. 462, 25. 463, 6. 473, 22. 23. 474, 39. Schliesslich hat auch die form des dialogs, die in tractat VI eine so erhebliche rolle spielt (vgl. 452, 6. 456. 457. 458. 459. 463. 464. 467. 468. 469. 470. 473. 474. 475) ein seitenstück in s. 126. Gegen die gleichsetzung bleibt freilich ein bedenken: in predigt XXXVII fehlt gänzlich das wort prïefen, das im tractat VI so häufig angewant wird, vgl. z. b. 448, 31. 32. 34. 449, 1, 33. 40. 450, 3. 5. 7. 26. $451,17.30$. 38. 39. 452,35 . 39. 453, 7. 454, 14. 26. 29. 37. 456, 36 . 37. 38. 459, 18. 462, 37. 467, 20. 468, 35. 469,15. 472,30. 474, 35 . 475,24 . 25. Und zwar finden sich diese beispiele sowol in den teilen, die der handschriftenklasse I angehören, als in denen, die nur in II überliefert sind und von Simon (in der s. 539 angeführten arbeit) als spätere einschaltungen betrachtet werden. 1)

1) Aus der tatsache, dass diese überschüsse von II auch selbständig vorkommen, ist kein schluss auf ihre unechtheit zu ziehen, namentlich nach 
Jundt no. 6 hat einmal hie sont wir verstan 254; im übrigen umfasst sie nur $1 \frac{1}{2}$ seiten, bietet also für die vergleichung $\mathbf{z u}$ wenig anhalt.

Die predigten XL. XLII. LXXX 'dagegen haben neben den formeln mit vizzen weder die wendungen mit verstan, noch die formel spriche ich me, noch dialogartiges. Dagegen zeigen sie, was in tractat VI ganz fehlt, versicherungspartikeln. Und zwar stimmen XLII ${ }^{1}$ ) und LXXX überein in der verwendung des seltenen bi guoter warheit 144, 36. 257, 17. 258, 1. 22, das sonst noch in XXXVII bégegnet $(281,1) .{ }^{2}$ )

Schliesslich werden die predigten $V$ und XLIX bei Pfeiffer, die predigt II bei Sievers, Hpt. 15,377, die predigten 8 und 9 bei Jundt und der tractat XVIII in beziehung gesetzt durch den eigentümlichen hinweis anf besonders häufig gebrauchte namen:

Pfeiffer 33,1 diu sint alliu in gote unde sint alliu in mir. Da endarf man weder Kuonvat noch Heinvich gedenken. - 33,5 swenie daz ich für ieman bite, für Heinrich oder für Kuonrat. - 163, 27 der ein bilde machen wil nach einem menschen, der machet ez niht na Kuonrate oder nach Ḧeinriche u. ff. -- Sievers 379, 85 he hiz alse wenic Gabriel alse Ciinrat. Jundt s. 259 wissent das da in der ainichait ist weder Chuonrat noch Heinvich. - 260 ich bitte nimer so wol won so ich nichtes nit bitte; und für nieman bitte, noch für Hainrichen, noch für Conraten. - Pfeiffer 591, 9 dar umbe bedarf ich niht gedenken, daz ich Heinirich oder Kuonrat noch Uolrich heize. - 592, 13 da heize ich ouch werlicher Kristus danne Johannes oder Jacob oder Uolrich.

Von diesen texten weist die predigt XLIX und die bei

dem, was wir jetzt durch Spamer über zersetzung und vererbung in mystikertexten wissen. Ehe man derartige ausscheidungen vornimmt, müsste doch vor allem geprüft werden, ob formale unterschiede zwischen dem echten und dem angeblich unechten bestehen. Und jețt würde die frage antwort verlangen, wie sich eine reihe von stücken ganz verschiedenen ursprungs unter sich und mit den 'echten' teilen in einer ganzen anzahl von eigentümlichkeiten zusammenfinden konnte (es sei dẹn, dass in den selbständigen fassungen starke abweichungen des textes vorlägen). Fällt die anschauung Simons über die einschaltungen, dann fällt auch das sonderbare, dass classe II zwar im textbestand schlechter, aber in den lesarten besser sein soll.

1) Pahncke s. 40 hatte die predigten XLII und XXV zusammengebracht; XXV zeigt aber weder eine formel mit wizzen, noch die wendung $b i$ guoter waitheit, dafür fünffaches wertiche $(97,25.98,4.19 .25 .26)$, das wider in XLII fehlt.

2) Mit guoter warheit in predigt VIII 45, 27 und 46, 30. 
Sievers und Jundt keine versicherungspartikeln auf: die predigt $\mathrm{V}$ bietet einmal werlich 32,18 ; der tractat XVIII mit seinen 15 seiten bietet folgendes: mit warkeit 584, 21; mit der varheit 584, 20; in rehter warheit 587,12; in der warheit 592,28; in warlheit 593, 1 ; werlich 591, 8. 592, 27. 28. 31. 36. 39; 593, 19. 31. Das reflexive sich verstan, das in tractat XVIII mehrfach angewant wird $(582,30.583,22.30 .31$. 584, 16. 23. 585, 16. 26 . 588,11 . 38. 593,22 ), fehlt in den drei predigten; allerdings findet es sich auch im tractat XVIII nur auf sechs der 15 seiten.

So viel dürfte unsere untersuchung ergeben haben: was in unseren ausgaben unter dem namen Eckhart geht, ist zum weitaus grössten teil herrenloses gut oder vielmehr gut einer ganzen reihe von verschiedenen unbekannten herren. ${ }^{1)}$ Meine versuche, die eigentümlichkeiten der von mir gekennzeichneten schriftstücke in anderweitigen denkmälern widerzufinden, haben bis jetzt zu keinem ergebnis geführt. Insbesondere zeigt der tractat, den Preger als eckhartisch veröffentlicht hat, Zs. f. historische theologie 1864, s. 163, keine der formalen eigentümlichkeiten von $\mathrm{V}$, VI oder XVII.

NS. Als ich das vorstehende schrieb, hatte mir die untersuchüng Spamers in der handschrift vorgelegen. Sie hat im druck mannigfache umgestaltung erfahren. So sind mir z.b. die ausführungen auf s. 343 und s. 396 zum teil unbekannt gewesen.

Ein wichtiger unterschied zwischen $V$ und $X V I I$ besteht noch beim substant. infinitiv. In $V$ adjectivische ergänżung nur bei einfachen verben $(422,4.422,16.433,26.444,8.447,39)$, die in der substantivierung vorausgiengen, bei denen also auch zuerst feste substantive entstanden (Zs. für deutsche wortf. 8, 329). In $V$ ferner keine ergänzung durch objectiven casus oder adverbiellen ausdruck; wol aber in XVII und hier adjectiv auch beim zusammengesetzten verbum. Belege: adj. + zusammengesetztes verbum: $548,27.549,36.550,12.14 .560,17.22 .565,21.571,26$. - Verbum + objectiver genetiv: einfaches 568,21; zusammengesetztes 554,14. 15. 562, 22. 571, 22. 574, 12. 17. - Verbum + adjectiv + objective oder adverbielle

1) Pahncke s. 49 fasst die predigten X்-XIV zu einer einheit zusammen. Aber $X$ hat viermal in der vowrheit: 54,14.16.21. 57,2; XI hat triuwen und in der warkeit, s. oben s. 547. Die drei andern haben werliche: XII: 61, 25 ; - XIII: 64, 18. 26. 65, 21. 34. 39. 66, 7. 9. 10. 67, 19 (neben gewerliche: 64, 32. 65, 40): - XIV 67,32. 70, 1. 4. 6. 22 (neben ir süllent wizzen 68,39 ). 
bestimmung: einfaches 548, 25. 26. 568, 1; zusammengesetztes 544, 12 . 548, 26. 549, 14. 550, 21. 558, 10. 11. 560, 8. 10. 562, 22. 571, 17 . Objectiver genetiv beim infinitiv ist auch jüngerer sprachgebrauch; damit dürfte wahrscheinlich werden, dass XVII jünger ist als V. ' Die predigt I (vgl. s. 547) stimmt auch in der behandlung des infinitivs zu XVII: vgl. 6, 8. 9. 35 . 7, 10. 13. 24. 8, 27. $(10,7)$.

GIESSEN, 21. mai 1908.

OTTO BEHAGHEL.

\section{ZUR DEUTSCHEN ETYMOLOGIE.}

I.

'Zur deutschen etymologie' hat Uhlenbeck 1901, Beitr. 26. 290-312 einen aufsatz geschrieben, in dessen mittelpunkt eigentlich mein Etymologisches wörterbuch der dentschen sprache zumal in der 1898 vollendeten 6. auflage steht. Es ist etwas spät, dass ich jetzt zu diesem aufsatz das wort ergreife und meinen von Uhlenbeck angegriffenen standpunkt verteidige. Meine verteidigung fällt mir heute viel leichter, als sie mir wol vor 7 jahren gewesen wäre. Damals hätte ich die polemik im wesentlichen mit eigenen waffen führen müssen, jetzt habe ich ganż unerwartet einen bundesgenossen erhalten, der für mich streiten kann. Ich meine den neuen Weigand, von dem bis jetzt, october 1908, die vier ersten hefte (A-Grille) vorliegen.

Ueber die etymologische seite der neuen auflage von Weigands Wörterbuch hat Uhlenbeck - im hinblick widerum auf mein Etymolog. wörterbuch - soeben in einer besprechung des Museums 1908, spalte 37 folgende äusserung: getan: 'In elk geval moet worden erkend, dat wij thans weer een goed etymologisch woordenboek van het Duitsch bezitten, dat op de hogte van den tijd is, iets wat sedert lang niet.meer van Kluge's werk mag gezegd worden.' Dieses urteil Uhlenbecks berechtigt mich, den etymologen des neuen Weigand mit der mehrzahl der einwendungen zu confrontieren, die Uhlenbeck Beitr. 26, 290 ff. gegen mich erhoben hat. 